\title{
Radiation Characteristics of a Quarter-Wave Monopole Antenna above Virtual Ground
}

\author{
Niazul Islam Khan, Anwarul Azim, and Shadli Islam
}

\begin{abstract}
In recent years, monopole antennas have become one of the vital components of cellular mobile communications and internet networks across the globe. Their relatively easy and low fabrication cost and faster installation makes them an obvious choice for developing countries. The most common form is the quarter-wave monopole, in which the antenna is approximately $1 / 4$ of a wavelength of the radio waves. In this paper, we present the analytical treatment of a quarter-wave monopole antenna above virtual ground. Virtual ground is realized by using bent radials around the vertical monopole element. Radiation resistance and gain of the quarter-wave monopole are mathematically derived and three-dimensional radiation patterns are simulated using sinusoidal current distribution. It is observed that for the case of the quarter-wave monopole, we achieve twice the gain of a dipole antenna of half-wave-length long. As the radiated power is limited to upper hemisphere of the ground plane, the total radiated power of the quarter-wave monopole is half of that of a dipole. Hence, the value of radiation resistance of the monopole is half of that of a dipole.
\end{abstract}

Index Terms - Ground plane, radiation pattern, directivity, half-wave dipole, monopole antenna.

\section{INTRODUCTION}

Monopole antenna above virtual ground, as the name itself indicates, is an antenna with a quarter wavelength long vertical radiator on a virtual ground plane. In telecommunication, a ground plane is a flat or nearly flat horizontal conducting surface that serves as part of an antenna, to reflect the radio waves from the other antenna elements. The plane does not necessarily have to be connected to the Earth [1]. Rather it can be realized virtually. The ground plane must have good conductivity because any resistance in the ground plane comes in series with the antenna. This series resistance causes power dissipation from the transmitter. In a quarter-wave monopole antenna above virtual ground, a near-perfect conducting ground plane is realized by several $1 / 4$ wavelength long radials as seen in Fig. 1. The feedline from the transmitter or receiver is connected between the bottom end of the radiator element and the virtually formed ground plane. So when current flows, there will also be radiation from the radials. As the radials are symmetrically arranged about the vertical element, the current in each radial

Manuscript received November 1, 2013; revised December 24, 2013.

Niazul Islam Khan and Anwarul Azim are with the Electrical and Electronic Engineering Department, East West University, Dhaka, Bangladesh (e-mail: niazulislam@ewubd.edu, azim@ewubd.edu).

Shadli Islam is with the Electrical and Electronic Engineering Department, Khulna University of Engineering and Technology, Khulna, Bangladesh (e-mail: ishad_29@yahoo.com). is flowing in an opposite direction (away from the center) to the current on the radial directly opposite to it and the total radiation in the horizontal plane will cancel. Thus a virtual conducting ground plane is formed. A typical feedline for the monopole antenna is a coaxial cable with its inner conductor connected through a hole to the ground plane to the vertical monopole element and its outer conductor connected by means of a flange to the ground plane [2]. Typically the inner conductor's diameter is equal to the monopole element's diameter and the outer conductor's diameter is equal to the ground plane hole diameter [2]. Impedance matching between the $50 \Omega$ coaxial cable feedline and the antenna feedpoint is achieved by bending the radials $45^{\circ}$ downwards with respect to the horizontal plane [3]. A monopole antenna above virtual ground with bent radius can be seen in Fig. 2 (a) with its current distribution shown in Fig. 2(b). Ground losses are avoided by elevating the antenna as higher as possible at least at quarter wavelength height from the earth's surface [2]. The other possibility is to add as many radials as possible in order to minimize the current on each radial. The current on each radial will be equal to the total current on the vertical element divided by the number of radials. So the guideline for this antenna is "the higher the better" about the elevation height and "the more the better" about the number of the radials [3]. Invented by the famous radio pioneer G. Marconi in 1895, it is sometimes called a Marconi antenna [4]. As being realized on a ground plane, it is also known as a ground plane antenna.



Fig. 1. Basic configuration of a monopole antenna above virtual ground. Symmetrically arranged four horizontal radials can be seen.

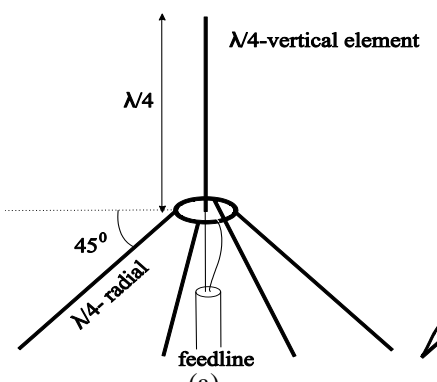

(a)

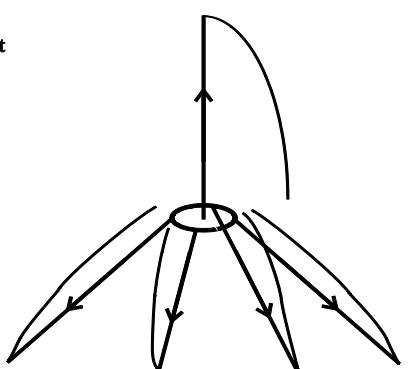

(b)
Fig. 2. (a) Radials of a monopole antenna above virtual ground are bent $45^{\circ}$ downwards with respect to the horizontal plane. (b) Curent distribution in a monopole antenna above virtual ground. 
Monopole antennas, having omni-directional radiation pattern characteristics, are very suitable for indoor applications, such as airplane, shopping center, hospital, etc. Particularly, their directivity on or near the radio horizon makes them suitable for communication systems where maximum operational range often depends on the directivity on the radio horizon [5]. Although designs for monopole antenna with coaxial cable involving double-folded [6] and triangular constructions [7] have been proposed in the past, those antennas only dealt with around 3/20 wavelength on similar impedance characteristics. The monopole antenna presented operates on 1/4 wavelength above virtual ground with its low angle radiation and minimized ground losses which makes them suitable for MIMO wireless communication systems [8]. Considering different operating frequencies especially in VHF and UHF bands, quarter-wave monopole offers several wide ranging advantages over dipole antennas. They are favored at frequencies above approximately $10 \mathrm{MHz}$ where the dimensions are manageable [9]. They are a good choice for VHF (very high frequency) and UHF (ultra high frequency) bands making them popular for FM broadcasting [10]. At VHF band ranging from 30 $\mathrm{MHz}$ to $300 \mathrm{MHz}$, a quarter-wave monopole can be $2.5 \mathrm{~m}$ (approximately $8 \mathrm{ft}$ ) long. At UHF band ranging from 300 $\mathrm{MHz}$ to $3 \mathrm{GHz}$, a quarter-wave whip is approx. $15 \mathrm{~cm}$ (6 in) long. For both cases, dipole antenna requires twice the length causing structural and placement limitations for various system designs.

Vertical monopole antennas above ground are also extensively used for amplitude modulated (AM) broadcasting in the frequency range $535-1605 \mathrm{kHz}$. For this application, monopole antennas are immensely helpful in reducing the required height [11]. They are also the antennas of choice for very low frequency (VLF) (3 to $30 \mathrm{kHz}$ ) and low frequency (LF) (30 to $300 \mathrm{kHz}$ ) communication systems. But for these applications, lower $\mathrm{h} / \lambda$ ratio deteriorates the radiation efficiency which can be improved by top shading [11]. At around $800 \mathrm{MHz}$ band, performance of quarter-wave monopole can be enhanced by stacking two monopoles around a phasing coil is used [12]. Open-ended monopole antennas are also used up to UHF and microwave frequencies where metallic conducting sheets, either solid or in the form of a screen, may be used instead of the ground to create the image antenna [11].

When ground is not available (such as in a vehicle) other metallic surfaces can serve as a ground plane (typically the vehicle's roof). Alternatively, radial wires placed at the base of the antenna can simulate a ground plane. For VHF bands, the radiating and ground plane elements can be constructed from rigid rods or tubes [13].

\section{AnAlytiCAl DEDUCTION OF THE RADiATION CHARACTERISTICS OF THE MONOPOLE}

We derive the properties of the monopole antenna above ground assuming a sinusoidal current distribution throughout the length of the antenna. As stated before, the radiation from the four radials cancels each other forming a virtual conducting ground plane. So the only radiating element is the
1/4 wavelength long vertical element. Therefore, the monopole antenna on the ground plane can be modeled as a half-wave dipole using the method of images [14]. In this case, the ground plane prevents monopole radiation into the hemisphere below the ground plane, but allows a radiation pattern identical to that of a dipole in the upper hemisphere [2]. Fig. 3 shows the quarter-wave monopole on the ground and its quarter-wave image forming a half-wave dipole. The bold dotted curved line represents the current distribution throughout the length of the antenna. We can write the expression for current distribution for the vertical elements as

$$
\tilde{I}=I_{0} \cos (\beta z)
$$

where $I_{0}$ is the maximum value of the current, and

$$
\beta=\text { phase constant }=\frac{2 \pi}{\lambda}
$$

At a point $P(r, \theta, \varphi)$ the magnetic vector potential is given by

$$
\vec{A}=\vec{A}_{Z}=\frac{\mu}{4 \pi} \int_{z=-\lambda / 4}^{\lambda / 4} \frac{\tilde{I}}{R} e^{-j \beta R} d z
$$

Substituting the value of $\tilde{I}$ in equation (2), the vector magnetic potential becomes,

$$
\vec{A}_{Z}=\frac{\mu I_{0}}{4 \pi} \int_{z=-\lambda / 4}^{\lambda / 4} \frac{\cos \beta z}{R} e^{-j \beta R} d z
$$

When the point $P(r, \theta, \varphi)$ is located far away from the antenna, we can write the equation $R \approx r-z \cos \theta$ to account for the phase shift and approximate it as $R \approx r$ for the distance in the denominator.

Moreover, we can use the following trigonometric formula

$$
\cos \beta z=\frac{e^{j \beta z}+e^{-j \beta z}}{2}
$$

to rewrite (3) as

$$
\vec{A}_{Z}=\frac{\mu I_{0}}{8 \pi r} e^{-j \beta r} \int_{z=-\lambda / 4}^{\lambda / 4}\left(e^{j \beta z}+e^{-j \beta z}\right) e^{j \beta z \cos \theta} d z
$$

After carrying out the whole calculation and required calculations, we reach the following expression for the z-component of magnetic vector potential

$$
\vec{A}_{Z}=\frac{\mu I_{0} e^{-j \beta r} \cos (\pi / 2 \cos \theta)}{2 \pi \beta r \sin ^{2} \theta}
$$

Now the magnetic field intensity in the radiation zone can be obtained using the following formula,

$$
\vec{H}=\frac{j \beta}{\mu} \sin \theta \vec{A}_{Z} \hat{a}_{\varphi}
$$

where $\hat{a}_{\varphi}$ is the unit vector along $\varphi$ direction. Substituting the expression of vector magnetic potential from (6) into (7), we obtain, 


$$
\vec{H}=\frac{j I_{0} e^{-j \beta r} \cos (\pi / 2 \cos \theta)}{2 \pi r \sin \theta} \hat{a}_{\varphi}
$$

The electric field intensity in the Fraunhofer zone can be obtained as,

$$
\vec{E}=\eta \vec{H} \hat{a}_{\theta}
$$

Here, $\hat{a}_{\theta}$ is the unit vector along $\theta$ direction and $\eta$ is the intrinsic impedance of the medium (air, in this case). Substituting the expression for the magnetic field from (8) into (9), we obtain the following as the expression of electric field,

$$
\vec{E}=\frac{j \eta I_{0} e^{-j \beta r} \cos (\pi / 2 \cos \theta)}{2 \pi r \sin \theta} \hat{a}_{\varphi}
$$

Using the expressions of magnetic and electric field intensity from (9) and (11), we obtain the expression for the average radiated power per unit area of the antenna as,

$$
\begin{aligned}
\langle\vec{S}\rangle & =\frac{1}{2} \operatorname{Re}\left|\vec{E} \times \vec{H}^{*}\right| \\
& =\frac{1}{2} \frac{|\vec{E}|^{2}}{\eta}
\end{aligned}
$$

After performing the necessary calculation, finally we reach to the following expression as,

$$
\begin{gathered}
\langle\vec{S}\rangle=\frac{\eta I_{0}^{2} \cos ^{2}\left(\frac{\pi}{2} \cos \theta\right)}{8 \pi^{2} r^{2} \sin ^{2} \theta} \\
=\frac{\eta I_{0}^{2}}{8 \pi^{2} r^{2}}\left(\frac{\cos \left(\frac{\pi}{2} \cos \theta\right)}{\sin \theta}\right)^{2}
\end{gathered}
$$

For a monopole antenna above virtual ground, the above expression is valid for the range $\mathrm{O} \leq \boldsymbol{\theta} \leq \frac{\pi}{2}$.

Using the following approximation [3]

$$
\left(\frac{\cos \left(\frac{\pi}{2} \cos \theta\right)}{\sin \theta}\right)^{2} \approx \sin ^{3} \theta
$$

which is valid for the range $0 \leq \theta \leq \pi$, we can write (12) as

$$
\langle\vec{S}\rangle=\frac{\eta I_{0}^{2}}{8 \pi^{2} r^{2}} \sin ^{3} \theta
$$

The total power radiated by the monopole is obtained by carrying out the following integration [7],

$$
\begin{aligned}
& P_{\text {rad }}=\oint_{S}\langle\vec{S}\rangle \cdot d \vec{S} \\
& =\int_{\theta} \int_{\phi}\langle\vec{S}\rangle r^{2} \sin \theta d \theta d \phi \\
& =r^{2} \int_{\theta=0}^{\pi / 2}\langle\vec{S}\rangle \sin \theta d \theta \int_{\phi=0}^{2 \pi} d \phi \\
& =\frac{\eta I_{0}^{2}}{8 \pi^{2}} \int_{\theta=0}^{\pi / 2} \sin ^{4} \theta d \theta \int_{\phi=0}^{2 \pi} d \phi
\end{aligned}
$$

After integration, we ultimately reach to the final form,

$$
P_{\text {rad }}=\frac{3 \eta I_{0}^{2}}{64}
$$

The radiation resistance is defined by the equation,

$$
P_{\text {rad }}=\frac{1}{2} I_{0}^{2} R_{\text {rad }}
$$

Using (15) and (16), we obtain the expression for radiation resistance for the quarter-wave monopole as,

$$
R_{\text {rad }}=\frac{3 \eta}{32}
$$

In free space, $\eta=120 \pi \Omega$. Inserting this value in (17), we get the value for radiation resistance for the monopole which is

$$
R_{\text {rad }}=35.343 \Omega
$$

which is one-half of a half-wave dipole antenna in free space.

Now we calculate the directive gain of the antenna using the following formula,

$$
G=\frac{4 \pi r^{2}\langle\vec{S}\rangle}{P_{r a d}}
$$

Substituting the values for $\langle\vec{S}\rangle$ and $P_{r a d}$ from (14) and (15), we decisively obtain the following equation of directive gain,

$$
G=\frac{32}{3 \pi} \sin ^{3} \theta
$$

Finally, the directivity of the monopole is found to be,

$$
D=\frac{32}{3 \pi}=3.39 \approx 5.31 \mathrm{~dB}
$$

Therefore, we deduce the antenna gain of a quarter-wave monopole antenna above virtual ground as $5.31 \mathrm{~dB}$ which is little bit more than twice the gain of a center-fed half-wave dipole antenna that is $2.16 \mathrm{~dB}$. Fig. 4 shows the radiation pattern of a quarter-wave monopole where the radiation is only limited to the upper hemisphere of the ground plane. Fig. 5 shows the corresponding radiation pattern of a half-wavelength dipole antenna where the radiation occurs in both the upper and lower hemisphere.

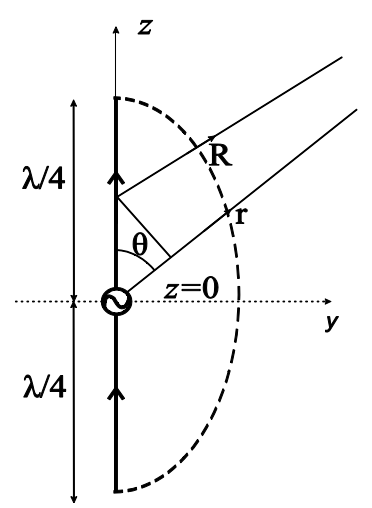

Fig. 3. Analysis of monopole antenna above virtual ground with sinusoidal current distribution/ 


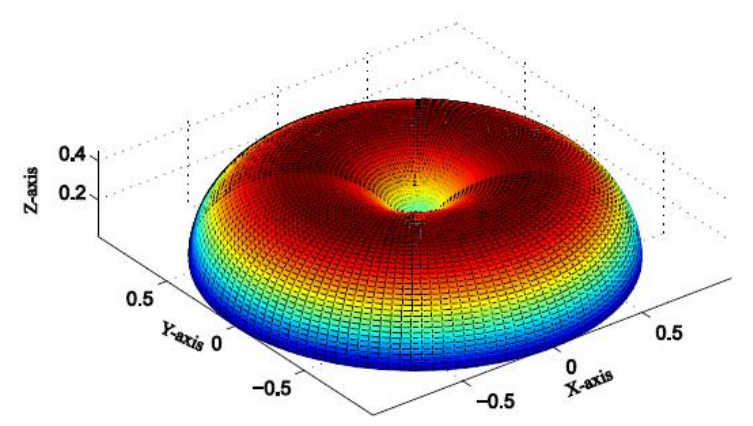

Fig. 4. Radiation pattern of a quarter-wave monopole antenna above virtual ground.

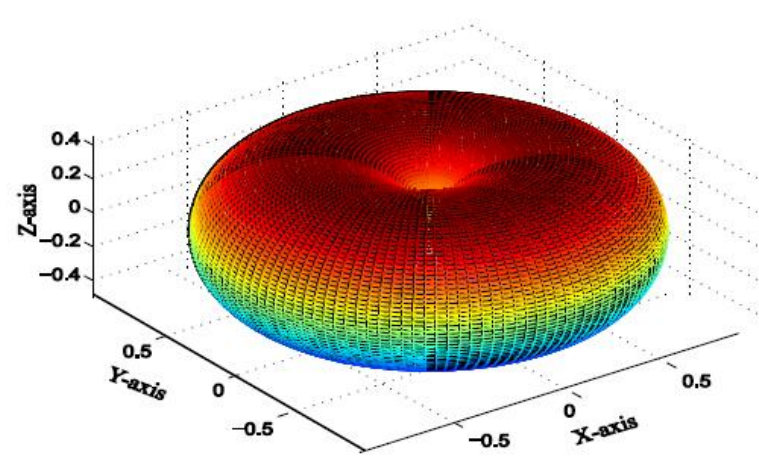

Fig. 5. Radiation pattern of a half-wave dipole antenna.

\section{CONCLUSION}

In this paper, we derived the analytical model of a quarter-wave monopole antenna above virtual ground. Directivity of $5.31 \mathrm{~dB}$ is calculated which is $3 \mathrm{~dB}$ better than that of a dipole antenna of half-wavelength long. The radiation pattern of the monopole is limited to the upper hemisphere of the ground plane. It is seen from the radiation pattern that maximum directivity is at the radio horizon which makes them a good choice for communication systems.

\section{ACKNOWLEDGMENT}

The authors would like to thank the Electrical and Electronic Engineering Department of East West University, Dhaka, Bangladesh for its support to make this work possible.

\section{REFERENCES}

[1] E. C. Latorilla, Practical Antenna Design: 140-150 MHz VHF Transceivers, $3^{\text {rd }}$ ed., Intellin Organization, LLC., 2006.

[2] M. M. Weiner, Monopole Antennas, Marcel Dekker, Inc., 2003.

[3] W. Fair Jr., Restricted space antennas. [Online]. Available: http://www.comportco.com/ w5alt/antennas/notes/antnotes.php?pg= 21

[4] T. Macnamara, Introduction to Antenna Placement \& Installation, John Wiley \& Sons, Inc, 2010.

[5] C. A. Ballanis, Antenna Theory: Analysis and Design, $2^{\text {nd }}$ ed., John Wiley \& Sons, Inc., 1997.
[6] T. Tsukiji, M. Yamasaki, and Y. Kumon, "Double-folded monopole antenna with coaxial cable," in Proc. Progress in Electromagnetics Research Symposium, 18-21 Aug. 2009, pp. 861-864.

[7] S. Lin, J. Qiu, X. Gu, H. Ren, and B. Zhao, "The simulation research of a novel uwb triangle monopole antenna," in Proc. 6th International Conference on Antenna Theory and Techniques, 17-21 Sept. 2007, pp. 256-258.

[8] C. Chiau, "Study of diversity antenna array for MIMO wireless communication systems," Ph.D. dissertation, Queen Mary University of London, UK, April 2006.

[9] M. A. Rahman, I. Amin, and N. I. Khan, "Design and fabrication of an FM broadcasting transmitter," Bachelor Thesis, Department of EEE, BUET, Dhaka, 2009.

[10] Ground-plane Antennas for 144, 222 and $440 \mathrm{MHz}$. [Online] Available: http://www.arrl.org/files/file/Technology/tis/info/pdf/ab18-16.pdf

[11] O. P. Gandhi, G. Lazzi, and C. M. Furse, Monopole Antennas. Encyclopedia of RF and Microwave Engineering, John Wiley \& Sons, Inc., 2005.

[12] W. A. Kissick, W. J. Ingram, J. M. Vanderau, and R. D. Jennings, Antenna System Guide, National Institute of Justice, April, 2001.

[13] S. Silver, Microwave Antenna Theory \& Design, Institution of Electrical Engineers, 1949.

[14] D. K. Cheng, Field and Wave Electromagnetics, $2^{\text {nd }}$ ed., Addison-Wesley Publishing Company Inc., 1983.

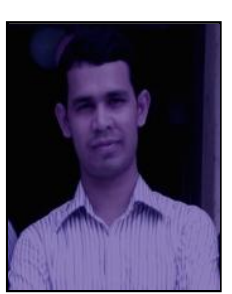

Niazul Islam Khan was born in Jhalakati, Bangladesh in 1987. He received his M.S. in Microelectronics (2012) from Ulm University, Ulm, Germany and B.S in Electrical and Electronic Engineering (2009) from BUET, Dhaka, Bangladesh. He worked as a research assistant in the Institute of Electron Devices and Circuits of Ulm University from May 2010 to March 2012. Currently, he is working as a lecturer in the Electrical and Electronic Engineering Department of East West University, Dhaka, Bangladesh. His research interest includes photonics, optoelectronics and fields and waves. He is a recipient of Deutschland stipend in 2011.

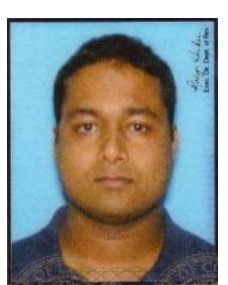

Anwarul Azim was born in Dhaka, Bangladesh. He obtained his B.Sc. in Electrical and Electronic Engineering (2006) from Islamic University of Technology, Gazipur, Bangladesh. He received the M.Sc. degree in Electrical Engineering (2010) from Colorado State University, USA. His main areas of interests are wireless networking and communications. $\mathrm{He}$ is currently working as a lecturer in the Electrical and Electronic Engineering Department of East West University, Dhaka, Bangladesh.

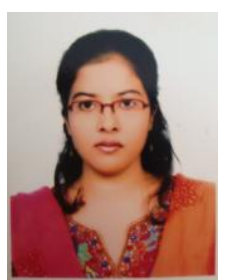

Shadli Islam was born in Dhaka, Bangladesh in 1992. She is an undergraduate student in the Electrical and Electronic Engineering Department of KUET, Khulna, Bangladesh. Her current research interests are in the field of biomedical engineering. At present, she is wokring on EMG signal analysis with the Electrical and Electronic Engineering Department of KUET. She won a gold medal as the best project award in the National Science Festival hosted by Viquarunnisa Noon Science Club in 2002 